\title{
Efecto de la concentración de inóculo y tiempo de fermentación en la producción de dextranos por Leuconostoc mesenteroides subsp. mesenteroides aislados de jugo de caña de azúcar.
}

\author{
Effect of concentration of inoculum and the fermentation time dextran production by \\ Leuconostoc mesenteroides subsp. mesenteroides isolated from sugar cane juice. \\ 1Juan Velásquez C. ${ }^{a}$ Heber Robles C. ${ }^{a}$
}

\section{RESUMEN}

Las variables ensayadas fueron: concentración de inóculo $5 \%, 10 \%$ y $15 \%$ y tiempo de fermentación 24,36 y 72 horas. El biorreactor fue alimentado con $250 \mathrm{~mL}$ de caldo hipersacarosado. La concentración del inóculo se determinó con la ayuda de la cámara de Newbahuer para luego estandarizarla a 1,6 × $10^{8} \mathrm{cel} / \mathrm{mL}$ en volúmenes de $5 \%, 10 \%$ y $15 \%$ constituyendo cada uno los inóculos definitivos. La eficiencia de la producción de dextranos fue calculada de acuerdo a la Concentración de dextrano $(\mathrm{g} / \mathrm{L})$, Productividad de dextrano $(\mathrm{g} / \mathrm{L} . \mathrm{h})$ y Conversión de sustrato (\%). La optimización de variables fue obtenida con la Prueba de Análisis de Varianza (ANAVA) y la Prueba Discriminatoria de Tukey. Las condiciones óptimas para la producción de dextranos por Leuconostoc mesenteroides subsp. mesenteroides fueron a partir de una concentración de inóculo de $5 \%$ y tiempo de fermentación de $36 \mathrm{~h}$, produciendo una concentración de dextrano de $30,730 \mathrm{~g} / \mathrm{L}$, productividad de $0,853 \mathrm{~g} / \mathrm{L}$.h y conversión de sustrato de 30,730\%.

Palabras clave: Dextrano, biorreactor, caldo hipersacarosado, eficiencia, productividad, optimización, inóculo.

\begin{abstract}
The variables tested were: inoculum concentration of $5 \%, 10 \%$ and $15 \%$ and fermentation time 24,36 and 72 hours. The bioreactor was fed with $250 \mathrm{~mL}$ of broth hipersacarosado. The inoculums concentration was determined with the help of the camera Newbahuer then standardized to $1,6 \times 10^{8} \mathrm{cel} / \mathrm{mL}$ in volumes of $5 \%, 10 \%$ and $15 \%$ are each final inocula. The efficiency of production of dextran was calculated according to the Concentration of dextran (g/L), Productivity of dextran (g/L.h) and substrate conversion (\%). The optimization of variables was obtained with the Test of Analysis of Variance (ANOVA) and Tukey discriminatory test. The optimum conditions for dextran production by Leuconostoc mesenteroides subsp. mesenteroides were from a concentration of $5 \%$ inoculums and fermentation time of $36 \mathrm{~h}$, producing a dextran concentration of $30,730 \mathrm{~g} / \mathrm{L}$, productivity of $0,853 \mathrm{~g} / \mathrm{L} . \mathrm{h}$ and substrate conversion of $30,730 \%$.
\end{abstract}

Keywords: Dextram, bioreactor, hipersacarosado broth, efficiency, productivity, optimization, inoculum.

\footnotetext{
'Carrera Profesional de Ingeniería Forestal y Ambiental, Universidad Nacional de Jaén.

${ }^{2}$ Facultad de Microbiología y Parasitología, Universidad Nacional de Trujillo.

a Biólogo.
} 


\section{INTRODUCCIÓN}

Dextrano $\left(\mathrm{C}_{6} \mathrm{H}_{10} \mathrm{O}_{5}\right)_{n}$ es un polisacárido de alto peso molecular, compuesto de unidades de D-glucosa unidos mediante enlaces glucosídicos $\alpha-(1-6)$; estructuralmente diversos y se caracterizan de acuerdo con el porcentaje, naturaleza y distribución de sus enlaces.

El dextrano se produce generalmente en cultivos de bacterias lácticas como Streptococcus, Acetobacter o Leuconostoc, en medios que contienen sacarosa; las células en crecimiento secretan una enzima inducible llamada dextransucrasa, que hidroliza la sacarosa, liberando fructosa y glucosa, esta última molécula sirve para sintetizar dextrano (Rodríguez y Hanssen, 2007).

El uso de los dextranos en la alimentación se debe principalmente a su capacidad para alterar las propiedades reológicas del agua presentes en los productos alimenticios, así como para modificar su textura. De hecho existe un gran número de dextranos que pueden ser utilizados como agentes espesantes o gelificantes, (Mata, 2006).

Existe una relevancia económica de productos generados industrialmente mediante procesos biotecnológicos, como el caso de antibióticos, ácidos orgánicos, aminoácidos, enzimas, polímeros y vitaminas, los cuales 0.2 billones \$/año son generados por polímeros, teniendo el dextrano hasta el año 2002 una producción en el mercado mundial de 200 toneladas por año (Kent y Riegel, 2007).

El proceso biosintético del dextrano es producido por la enzima dextransucrasa DSRE, que cataliza la transferencia de glucosa proveniente de la sacarosa a moléculas aceptoras, principalmente azúcares, para la síntesis de glucooligosacáridos.

En ausencia de aceptores, el principal producto de la reacción es la dextrana, un polímero compuesto por enlaces $\alpha-(1-6)$ en la cadena principal y con ramificaciones en $\alpha-(1-2), \alpha-(1-3)$ y $\alpha-(1-4)$ (Domínguez et al. 2004).

(Qaner et al. 2001) llegaron a producir con la cepa Leuconostoc mesenteroides Pcsir-3 un dextrano distinto al producido por la cepa industrial Leuconostoc mesenteroides NRRLB-512F, en un tiempo mínimo de fermentación de 12 horas.

Pinchi y Esparza, (2004) investigaron la producción de dextranos por Leuconostoc mesenteroides CCBB 017 a diversos tiempos de fermentación, reactivando la cepa a $28{ }^{\circ} \mathrm{C}$ y pH 7,0 por 24 horas, logrando obtener un inóculo equivalente a $10^{\circ} \mathrm{cel} / \mathrm{L}$.

Después de iniciado el proceso fermentativo en tanque aireado y agitado a $22{ }^{\circ} \mathrm{C}+/-1,100 \mathrm{~g} / \mathrm{L}$ de sacarosa, $\mathrm{pH} 7,0,0,5$ vvm y $600 \mathrm{rpm}$; deteniéndose la producción en cada uno de los biorreactores a 5, 10, $15,20,30,40$ y 50 horas de fermentación, se obtuvo luego de precipitar con etanol $\left(96^{\circ}\right)$ y $2 \mathrm{~mL} \mathrm{KCl}$ producciones de dextrano de $3,86 \mathrm{~g} / \mathrm{L}$ en $5 \mathrm{~h} ; 8.43 \mathrm{~g} / \mathrm{L}$ en $10 \mathrm{~h} ; 13.94 \mathrm{~g} / \mathrm{L}$ en $15 \mathrm{~h} ; 18.18 \mathrm{~g} / \mathrm{L}$ en $20 \mathrm{~h} ; 22.05$ $\mathrm{g} / \mathrm{L}$ en $30 \mathrm{~h} ; 23.18 \mathrm{~g} / \mathrm{L}$ en $40 \mathrm{~h}$ y $24.63 \mathrm{~g} / \mathrm{L}$ en $50 \mathrm{~h}$ respectivamente.

El presente trabajo de investigación presentó los siguientes objetivos: aislar e identificar una cepa de Leuconostoc mesenteroides subsp. mesenteroides a partir del jugo de caña de azúcar y determinar el efecto de la concentración de inóculo y tiempo de fermentación en la producción de dextranos por Leuconostoc mesenteroides subsp. mesenteroides.

\section{MATERIALES Y MÉTODOS}

\section{Métodos}

\section{Variables en estudio:}

Variables independientes

$\mathrm{A}=$ Concentración de inóculo $(\%) \quad 5,10,15$

$\mathrm{B}=$ Tiempo de fermentación (horas) $24,36,72$

\section{Variable dependiente}

$\mathrm{C}=$ Producción de dextranos $(\mathrm{g} / \mathrm{L})$ 


\section{Diseño Experimental}

Se aplicó el Diseño Factorial $3 \times 3$ para estimar las diferencias significativas del efecto de las variables sobre la producción de dextranos para lo cual se efectuó 09 ensayos con 03 repeticiones (Tabla 1 y Tabla 2).

Tabla 1. Diseño experimental verdadero (Diseño factorial $3 \times 3$ )

\begin{tabular}{ccccc}
\hline Variables & \multicolumn{3}{c}{ Tiempo (h) } \\
\hline Concentración & A1 & A1 B1 & A1 B2 & A1 B3 \\
Inoculo & A2 & A2 B1 & A2 B2 & A2 B3 \\
$(\%)$ & A3 & A3 B1 & A3 B2 & A3 B3 \\
\hline
\end{tabular}

Ai,Bi: concentración de inóculo versus tiempo de fermentación.

Tabla 2. Diseño de las variables establecidas y ensayadas

\begin{tabular}{cccc}
\hline Variables & $\mathbf{2 4} \mathbf{h}$ & $\mathbf{3 6} \mathbf{h}$ & $\mathbf{7 2} \mathbf{h}$ \\
\hline $\mathbf{5 \%}$ & $5 \% 24 \mathrm{~h}$ & $5 \% 36 \mathrm{~h}$ & $5 \% 72 \mathrm{~h}$ \\
$\mathbf{1 0} \%$ & $10 \% 24 \mathrm{~h}$ & $10 \% 36 \mathrm{~h}$ & $10 \% 72 \mathrm{~h}$ \\
$\mathbf{1 5 \%}$ & $15 \% 24 \mathrm{~h}$ & $15 \% 36 \mathrm{~h}$ & $15 \% 72 \mathrm{~h}$ \\
\hline
\end{tabular}

\section{Evaluación de la Producción de Dextranos}

\section{A. Fase Pre Fermentativa}

\section{Aislamiento e identificación de Leuconostoc mesenteroides subsp. mesenteroides.}

Se tomó una alícuota de $1 \mathrm{~mL}$ de jugo de caña de azúcar y se procedió a realizar diluciones en tubos que contenían $9 \mathrm{~mL}$ de solución salina fisiológica hasta llegar a $10^{-2}$, para luego tomar de la última dilución $0,1 \mathrm{~mL}$ y sembrarlo por duplicado mediante la técnica de agotamiento y estría en agar hipersacarosado en placas de Petri. Las placas fueron incubadas a $28^{\circ} \mathrm{C}$ durante 48 horas. (Tabla 1 y Tabla 2).
Se seleccionó la colonia que presentó mayor diámetro de goma incolora y características macroscópicas como tamaño de colonia, forma puntiforme, aspecto opaco y elevación convexa. Después se repicó en viales conteniendo agar hipersacarosado modificado e incubados a $28{ }^{\circ} \mathrm{C}$ por 48 horas y conservados en refrigeración. (Tabla 1 y Tabla 2 ).

Leuconostoc mesenteroides subsp. mesenteroides fue identificada en función de las características morfológicas y fisiológicas, como prueba de la catalasa, formación de ácidos a partir de carbohidratos (glucosa, fructosa, sucrosa, arabinosa y trehalosa) y formación de dextranos a partir de sacarosa (Holt et al. 1994). 
Los biorreactores tipo tanque aireado agitado, estuvieron constituidos por frascos de vidrio de un litro de capacidad cuyo extremo superior estuvo cubierto por una tapa de goma hermética que presentó dos orificios. A través del primero de ellos se ingresó una cánula de plástico taponada en su extremo exterior con algodón para permitir la salida de gases y a través del segundo de los orificios se ingresó un difusor de aire generado por una bomba Élite 803 de 4,0 watts $(0,5 \mathrm{vvm})$ y esterilizado por un sistema de burbujco en solución de cloruro de sodio al $20 \%$. (Villanueva y Carreño, 2005).

El cultivo puro de Leuconostoc mesenteroides subsp. mesenteroides fue reactivada en una placa de petri con agar hipersacarosado a $28^{\circ} \mathrm{C}$ durante 24 horas y del crecimiento obtenido se sembró en otra placa de petri con agar hipersacarosado modificado mediante la técnica de agotamiento y estría.

De la biomasa obtenida se obtuvo una colonia característica para obtener una suspensión de células en solución salina físiológica estéril y se inoculó $3 \mathrm{~mL}$ en un matraz conteniendo $30 \mathrm{~mL}$ de caldo hipersacarosado modificado.

El matraz fue incubado en agitación constante a $28^{\circ} \mathrm{C}$ durante 12 horas para luego llevar el contenido a un matraz con $300 \mathrm{~mL}$ de caldo hipersacarosado modificado. Enseguida fue incubado a $28{ }^{\circ} \mathrm{C}$ durante 12 horas, para luego determinar la concentración celular con la ayuda de la cámara de Neubahuer y estandarizar a 1,6 $\mathrm{x}$ $10^{8} \mathrm{cel} / \mathrm{mL}$ en volúmenes de $5 \%, 10 \%$ y $15 \%$ constituyendo cada uno los inóculos definitivos.

\section{B. Fase Fermentativa}

A los nueve biorreactores tipo tanque aireado agitado de $1 \mathrm{~L}$ de capacidad se le agregó $250 \mathrm{~mL}$ de caldo hipersacarosado, a razón de tres biorreactores por sistema a ensayar.Luego se inoculó la concentración celular estandarizada $\left(1,6 \times 10^{8} \mathrm{cel} / \mathrm{mL}\right)$ en volúmenes de $5 \%, 10 \%$ y $15 \%$, se homogenizó y se incubaron a $28{ }^{\circ} \mathrm{C}$ durante los tiempos de fermentación discñados 24 h, 36 hy 72 h.

\section{Fase Post Fermentativa}

En frascos de vidrio de $500 \mathrm{~mL}$ de capacidad se colocó $100 \mathrm{~mL}$ de caldo hipersacarosado fermentado y se añadió $9 \mathrm{~mL}$ de cloruro de sodio al $2 \%$ y $200 \mathrm{~mL}$ de isopropanol de $90^{\circ}$ y $3 \mathrm{~mL}$ de etanol absoluto. Después de un reposo de 30 minutos el material fue centrifugado a $8000 \mathrm{rpm}$ durante 15 minutos y el sedimento se coloco sobre papel aluminio y llevado a la estufa a $50^{\circ} \mathrm{C}$ durante 48 horas y así obtener el dextrano seco. Luego se peso en una balanza analítica y se determino el peso seco respectivo.

\section{RESULTADOS}

Producción de dextranos por efecto de la concentración de inóculo y tiempo de fermentación por Leuconostoc mesenteroides subsp. mesenteroides.

En la Tabla 3 se presentan los valores obtenidos de la producción de dextranos expresados en promedios de peso seco de los 09 tratamientos con sus repeticiones, teniendo a la concentración de inóculo $5 \%$ y tiempos de fermentación de 36 h y 72 h como los más elevados con $30,73 \mathrm{~g} / \mathrm{L}$ y $29,80 \mathrm{~g} / \mathrm{L}$. Asimismo los valores de concentración de inóculo $10 \%$ y tiempo de fermentación 24 h como los de menor rendimiento con $14,27 \mathrm{~g} / \mathrm{L}$ respectivamente. 
Tabla 3. Producción de dextranos: peso seco, por efecto de la concentración de inóculo y tiempo de fermentación.

\begin{tabular}{|c|c|c|c|}
\hline $\begin{array}{c}\text { Concentración } \\
\text { de inóculo } \\
(\%)\end{array}$ & $\begin{array}{c}\text { Tiempo } \\
\text { (h) }\end{array}$ & $\begin{array}{l}\text { Peso } \\
\text { seco } \\
(\mathrm{g} / \mathrm{L})\end{array}$ & $\begin{array}{l}\text { Promedio } \\
\text { Peso } \\
\text { seco }(\mathrm{g} / \mathrm{L})\end{array}$ \\
\hline 5 & 24 & 13,68 & \\
\hline 5 & 24 & 15,16 & 14,57 \\
\hline 5 & 24 & 14,83 & \\
\hline 5 & 36 & 35,08 & \\
\hline 5 & 36 & 25,87 & 30,73 \\
\hline 5 & 36 & 31,22 & \\
\hline 5 & 72 & 30,09 & \\
\hline 5 & 72 & 29,18 & 29,80 \\
\hline 5 & 72 & 30,15 & \\
\hline 10 & 24 & 12,92 & \\
\hline 10 & 24 & 14,65 & 14,27 \\
\hline 10 & 24 & 15,24 & \\
\hline 10 & 36 & 20,31 & \\
\hline 10 & 36 & 17,85 & 18,88 \\
\hline 10 & 36 & 18,49 & \\
\hline 10 & 72 & 29,00 & \\
\hline 10 & 72 & 24,97 & 25,84 \\
\hline 10 & 72 & 23,56 & \\
\hline 15 & 24 & 19,17 & \\
\hline 15 & 24 & 18,59 & 18,45 \\
\hline 15 & 24 & 17,58 & \\
\hline 15 & 36 & 18,58 & \\
\hline 15 & 36 & 15,56 & 16,03 \\
\hline 15 & 36 & 13,96 & \\
\hline 15 & 72 & 19,17 & \\
\hline 15 & 72 & 16,31 & 17,50 \\
\hline 15 & 72 & 17,03 & \\
\hline
\end{tabular}


En la Figura 1, se observa los valores de concentración de inóculo $(5 \%, 10 \%$ y $15 \%)$ y tiempos de fermentación (24h, $36 \mathrm{~h}$ y $72 \mathrm{~h}$ ), correlacionados con la producción de dextranos en función de concentración $(\mathrm{g} / \mathrm{L})$, manifestando que la mayor concentración de dextranos se obtiene con una concentración de inóculo de 5\% y el mejor tiempo de fermentación es de $36 \mathrm{~h}$ y $72 \mathrm{~h}$, obteniendo concentraciones máximas de dextranos de $30,73 \mathrm{~g} / \mathrm{L}$,

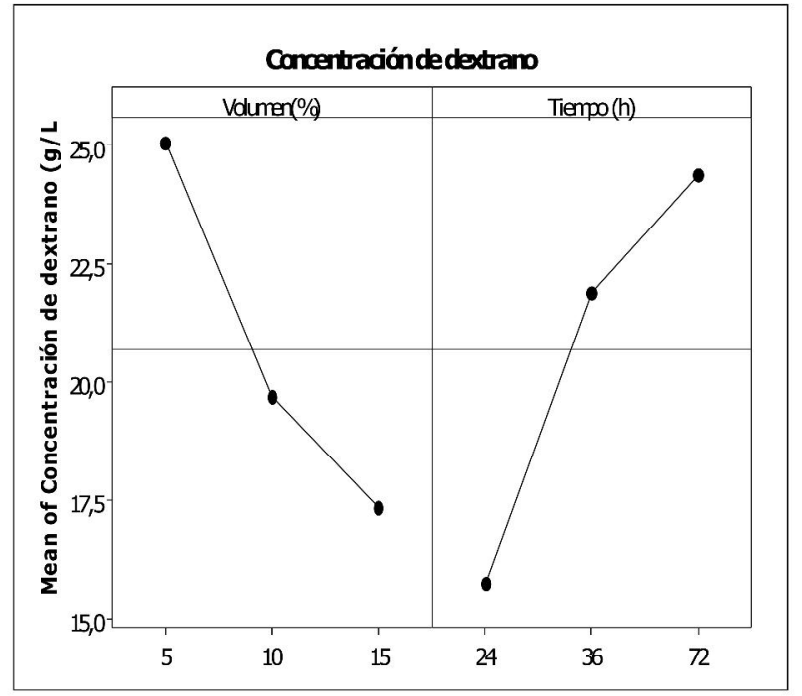

Figura 1. Valores de concentración de inóculo (volumen \%) y tiempo de fermentación (h) encontrados para la producción de dextrano (concentración).

En la Figura 2, los valores encontrados entre las interacciones de concentración de inóculo $(5 \%$,
$10 \%$ y $15 \%)$ y tiempos de fermentación $(24 \mathrm{~h}$, $36 \mathrm{~h}$ y $72 \mathrm{~h}$ ) para la producción de dextranos ( $\mathrm{g} / \mathrm{L})$, manifiesta que con una concentración de inóculo de $5 \%$ y un tiempo de fermentación de $36 \mathrm{~h}$, se obtiene una concentración de dextranos máxima de $30,73 \mathrm{~g} / \mathrm{L}$. Lo cual constituye que las variables independientes como concentración de inóculo y tiempos de fermentación tienen una significación directa en la producción del biopolímero dextrano.

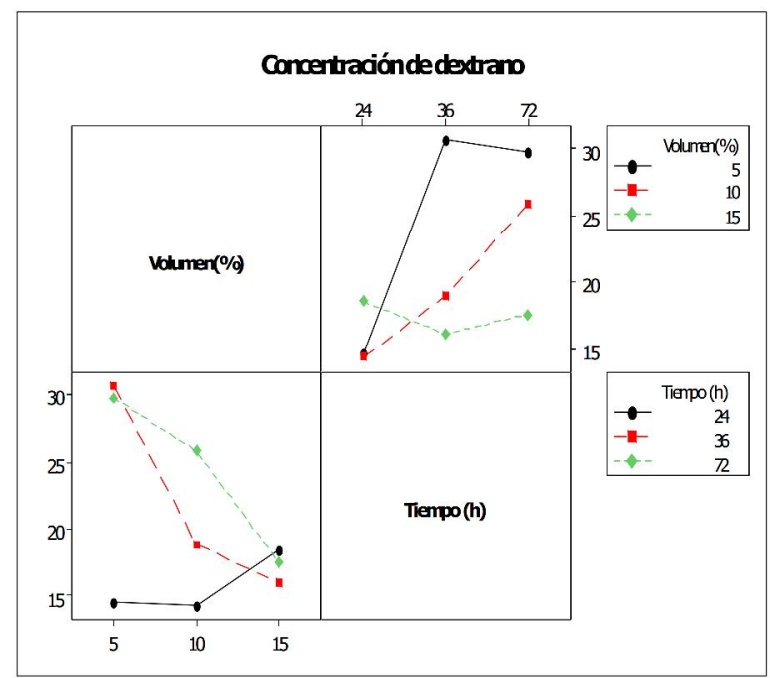

Figura 2. Valores encontrados entre las interacciones de concentración de inóculo (volumen \%) y tiempos de fermentación (h) para la producción de dextranos (concentración).

En la Tabla 4 se presentan los valores de rendimiento de dextrano expresado en concentración de dextrano $(\mathrm{g} / \mathrm{L})$, productividad (g/L.h) y conversión de dextrano (\%) teniendo los siguientes resultados:

La concentración de dextrano máxima $(\mathrm{g} / \mathrm{L})$ que se obtuvo a la concentración de inóculo 5 \% y tiempo de fermentación de $36 \mathrm{~h}$ fue de $30,730 \mathrm{~g} / \mathrm{L}$ y 14,272 g/L como el valor mínimo a una concentración de inóculo de $10 \%, 24 \mathrm{~h}$ de fermentación.

La productividad fue de $0,853 \mathrm{~g} / \mathrm{L}$.h como el valor más elevado correspondiente a la variable $5 \%, 36$ h y el valor más bajo fue de $0,243 \mathrm{~g} / \mathrm{L}$.h a $15 \%$ y $72 \mathrm{~h}$; la conversión de sustrato fue de $30,730 \%$ como el valor más elevado correspondiendo a $5 \%, 36$ h y 14,272 \% como la conversión más baja con $10 \%$ de inóculo y 24 h de fermentación. 
Tabla 4. Rendimiento promedio de dextrano: concentración de dextrano, productividad y conversión de sustrato por efecto de la concentración de inóculo y tiempo de fermentación.

\begin{tabular}{ccccc}
\hline $\begin{array}{c}\text { Concentración de } \\
\text { inóculo } \\
(\%)\end{array}$ & $\begin{array}{c}\text { Tiempo } \\
\text { (h) }\end{array}$ & $\begin{array}{c}\text { Rendimiento Promedio } \\
\text { Concentración } \\
\text { de dextrano } \\
(\mathbf{g} / \mathbf{L})\end{array}$ & $\begin{array}{c}\text { Productividad } \\
\mathbf{( g / L . h})\end{array}$ & $\begin{array}{c}\text { Conversión de } \\
\text { sustrato (\%) }\end{array}$ \\
\hline 5 & 24 & 14,559 & 0,606 & 14,559 \\
5 & 36 & 30,730 & 0,853 & 30,730 \\
5 & 72 & 29,808 & 0,414 & 29,808 \\
10 & 24 & 14,272 & 0,594 & 14,272 \\
10 & 36 & 18,887 & 0,524 & 18,887 \\
10 & 72 & 25,849 & 0,359 & 25,849 \\
15 & 24 & 18,451 & 0,768 & 18,451 \\
15 & 36 & 16,039 & 0,445 & 16,039 \\
15 & 72 & 17,508 & 0,243 & 17,508 \\
\hline
\end{tabular}

Evaluación de la producción de dextranos por efecto de la concentración de inóculo y tiempo de fermentación.

La prucba de " $\mathrm{F}$ " del análisis de varianza para el factorial $3 \times 3$ evidenció significación para los tratamientos, concentración de inóculo (A), tiempo de fermentación (B) e interacción AxB. La significación demuestra que existe efecto de las variables estudiadas en la producción de dextrano a partir de Leuconostoc mesenteroides subsp. mesenteroides (Tabla 5).

Tabla 5. Análisis de varianza de los valores de concentración de dextrano por efecto de la concentración de inóculo y tiempo de fermentación.

\begin{tabular}{lccccc}
\hline Fuente & $\begin{array}{c}\text { Suma de cuadrados } \\
\text { Tipo III }\end{array}$ & GL & Media cuadrática & F & P \\
& 12533.220 & 9 & 1392,580 & 300,555 & 0,000 \\
Modelo & & & & & \\
Concentración & 280,529 & 2 & 140,26 & 30,273 & 0,000 \\
de inoculo (A) & 354,605 & 2 & 177,303 & 38,267 & 0,000 \\
Tiempo (B) & 352,897 & 4 & 88,224 & 19,041 & 0,000 \\
AxB & 83,400 & 18 & 4,633 & & \\
Error & $\mathrm{R}-\mathrm{Sq}(\mathrm{adj})=88,76 \%$ & & & \\
\hline $\mathrm{S}=2,15252$ & & & & &
\end{tabular}


La prueba discriminatoria de Tukey $(\alpha=0,05)$ para concentración de dextrano evidencia que la interacción concentración de inóculo con tiempo de fermentación $10 \%, 72 \mathrm{~h} ; 5 \%, 72 \mathrm{~h}$ y $5 \%, 36 \mathrm{~h}$ presenta diferencias estadísticas referentes a los demás tratamientos (Tabla 6).

labla 6. Prueba discriminatoria de lukey $(\alpha=0,05)$ de la concentración de dextrano por efecto de la interacción concentración de inóculo con tiempo de fermentación.

\begin{tabular}{|c|c|c|c|}
\hline \multirow[t]{2}{*}{ Tratamientos } & \multirow[t]{2}{*}{$\mathbf{N}$} & \multicolumn{2}{|c|}{ Subconjuntos } \\
\hline & & 1 & 2 \\
\hline $10 \%-24 h$ & 3 & 14,272667 & \\
\hline $5 \%-24 h$ & 3 & 14,559667 & \\
\hline $15 \%-36 h$ & 3 & 16,039000 & \\
\hline $15 \%-72 h$ & 3 & 17,508333 & \\
\hline $15 \%-24 h$ & 3 & 18,451667 & \\
\hline $10 \%-36 h$ & 3 & 18,887333 & \\
\hline $10 \%-72 \mathrm{~h}$ & 3 & & 25,849000 \\
\hline $5 \%-72 h$ & 3 & & 29,808667 \\
\hline $5 \%-36 h$ & 3 & & 30,730000 \\
\hline Significación & & 0,242 & 0,189 \\
\hline
\end{tabular}

\section{DISCUSIÓN}

En el Perú, dextrano o dextran es empleado en la industria farmacéutica, minera y especialmente en la alimentaria, como espesante o gelificante, mejorando sus caracteres organolépticos y estructurales (Mata, 2006). Como consecuencia de la actividad fermentativa de los microorganismos, como Streptococcus, Acetobacter, Leuconostoc y algunas cepas de hongos como Penicillium aculeatum (Erhardt et al. 2008) la producción de dextranos se proyecta a ser uno de los biopolímeros con mayor aplicación en beneficio de la industria.

Varias metodologías y técnicas han sido diseñadas, teniendo como factores determinantes a la temperatura, $\mathrm{pH}$, tiempos de fermentación y muy sensiblemente el diseño y formulación del medio de cultivo (Hamdy et al. 1954). En el presente trabajo, la sacarosa en un $10 \%$ sirvió como sustrato para la producción del dextrano, además de extracto de levadura $5 \mathrm{~g}$, triptona $10 \mathrm{~g}$, citrato sódico $1 \mathrm{~g}$, glucosa $5 \mathrm{~g}$, gelatina $2,5 \mathrm{~g}$, agar-agar $15 \mathrm{~g}$ y agua destilada $1000 \mathrm{~mL}$, lo cual permite comparar con los medios de cultivo utilizados por (Qader et al. 2006) que agregó una fuente de nitrógeno suplida con diferentes sales minerales, teniendo a Leuconostoc mesenteroides PCSI-4 como la de mayor producción con 65 g/L durante $49 \mathrm{~h}$. 
(Rodriguez y Hanssen, 2007) utilizó residuos agroindustriales como cáscara de naranja, piña y cachaza de caña papelera como sustratos, teniendo a las cáscaras de naranja como la de mejor producción con $3,4 \mathrm{~g} / \mathrm{L}$ conteniendo en su medio entre $2 \%$ - $5 \%$ de sacarosa.

(Behravan et al. 2003) del centro de investigación biotecnológica de Irán realizó una investigación sobre la producción de dextrano a partir de fuentes económicas del país, como melazas de remolacha azucarera y extracto de salvado de trigo, logrando una concentración de 9,44 g/L de dextrano y (Lopretti, 2002) realizó un proyecto sobre la valorización de residuos para la generación de productos de alto valor agregado, entre ellos los polisacáridos, utilizando agua de coco, jugo de cáscaras de piña, naranja, mandarina, uvas, entre otros, con concentraciones entre $2,5 \mathrm{~g} / \mathrm{L} \mathrm{y} 8 \mathrm{~g} / \mathrm{L}$ de dextrano.

La producción de dextranos expresado como concentración de dextrano $(\mathrm{g} / \mathrm{L})$, productividad de dextrano $(\mathrm{g} / \mathrm{L} . \mathrm{h})$ y conversión de sustrato $(\%)$ en el presente trabajo, ha permitido evaluar los factores de concentración de inóculo, presentado volúmenes al 5 $\%, 10 \%$ y $15 \%$ y tiempos de fermentación de $24 \mathrm{~h}, 36$ h y $72 \mathrm{~h}$, llegando a obtener resultados muy buenos que permitirán optimizar la obtención de este biopolímero, ya que mejorando estas variables podría representar una disminución en los costos de consecución de materia prima para los bioprocesos de obtención de dextranos.

Se utilizó a Leuconostoc mesenteroides subsp. mesenteroides aislada del jugo de la caña de azúcar de la empresa Azucarera Agroindustrial Pomalca, para ser cultivado en agar hipersacarosado en un biorreactor aireado agitado con un volumen de 250 $\mathrm{mL}$. La concentración de dextrano $(\mathrm{g} / \mathrm{L})$ tuvo como valor máximo un $30,730 \mathrm{~g} / \mathrm{L}$ a partir de la interacción entre $5 \%$ y 36 h y como valor mínimo un $14,272 \mathrm{~g} / \mathrm{L}$ correspondiendo a $10 \%$ y $24 \mathrm{~h}$ respectivamente, mejorando el trabajo realizado por (Pinchi y Esparza, 2004) que obtuvo dextranos de Leuconostoc mesenteroides CCBT-017 a diferentes tiempos de fermentación usando biorreactores tipo tanque aireado agitado e inóculo de $10^{9} \mathrm{cel} / \mathrm{mL}$ concentraciones de $3,86 \mathrm{~g} / \mathrm{L}$ en $5 \mathrm{~h} ; 8,43 \mathrm{~g} / \mathrm{L}$ en $10 \mathrm{~h}$; $13,94 \mathrm{~g} / \mathrm{L}$ en $15 \mathrm{~h} ; 18,18 \mathrm{~g} / \mathrm{L}$ en $20 \mathrm{~h} ; 22,05 \mathrm{~g} / \mathrm{L}$ en 30 h; 23,18 g/Len 40 hy $24,63 \mathrm{~g} / \mathrm{La} 50 \mathrm{~h}$.

Asimismo se aproxima a los resultados que obtuvieron (Qader et al. 2006) con una producción de dextranos entre $36 \mathrm{~g} / \mathrm{L}$ y $44 \mathrm{~g} / \mathrm{L}$ durante un tiempos de $18 \mathrm{~h}$ a $48 \mathrm{~h}$ a partir de Leuconostoc mesenteroides PCSIR-4. Sin embargo, existen cepas de Leuconostoc mesenteroides subsp. mesenteroides que han producido concentraciones elevadas de hasta $70 \mathrm{~g} / \mathrm{L}$ durante $18 \mathrm{~h}$ con sustratos de sacarosa de $200 \mathrm{~g} / \mathrm{L}$ (Landon et al. 1993) y (Vedyashkina et al. 2004) optimizaron la producción de dextrano suplementando su medio de cultivo con $17,5 \%$ de sacarosa, $\mathrm{pH}$ inicial de 6,75 y melaza, obteniendo concentraciones entre $54 \mathrm{~g} / \mathrm{L}$ y $55 \mathrm{~g} / \mathrm{L}$ de dextrano.

Muy cercanos a estos datos y como una fuente alternativa a la producción de dextranos esta la utilización de micelios de Penicillium aculeatum que produce rendimientos de $20 \mathrm{~g} / \mathrm{L}$ en un máximo de $72 \mathrm{~h}$ (Erhardt et al. 2008). Los tiempos de fermentación (h) aplicados para la producción de dextrano en el presente trabajo, $24 \mathrm{~h}, 36 \mathrm{~h}$ y $72 \mathrm{~h}$, enfrentados a los concentraciones de inóculo (\%) concuerdan con los ensayados por (Qader et al. 2006) en la cual menciona que las concentraciones de inóculos bajas necesitan pasar por una fase de latencia que sintetice sus estructuras básicas y entrar a la fase logarítmica de rápido crecimiento en biomasa y formación de productos a finales de la misma (dextranos), teniendo como consecuencia de la autólisis de las células por efecto de endoenzimas secretadas al medio de cultivo.

Finalmente, el trabajo realizado tubo como concentración de inóculo (\%) y tiempos de fermentación (h) óptimos para la producción de dextranos a $5 \%$ y $36 \mathrm{~h}$, respectivamente, permitiendo manejar variables de concentración de dextrano $(\mathrm{g} / \mathrm{L})$, productividad $(\mathrm{g} / \mathrm{h})$ y conversión de sustrato $(\%)$ en forma eficazy eficiente. 


\section{CONCLUSIONES}

- Se aisló un cultivo puro de Leuconostoc mesenteroides subsp. mesenteroides a partir de jugo de caña de azúcar de la Cooperativa Agroindustrial Azucarera Pomalca, Región Lambayeque.

- Con la concentración de inócula 5\% y tiempos de fermentación de 36 h utilizando Leuconostoc mesenteroides subsp. mesenteroides se obtuvo una producción máxima de dextranos de 30,730 $\mathrm{g} / \mathrm{L}$; una productividad de 0,853 g/L.h y una conversión de sustrato de $30,730 \%$.

- La prueba F del análisis de varianza (ANAVA) y prueba discriminatoria de TUKEY, determinaron que existe diferencias significativas y estadísticas para los tratamientos ensayados entre concentración de inóculo (\%) y tiempos de fermentación (h).

- El efecto de la concentración de inóculo (\%), tiempos de fermentación (h), demostró que es posible obtener dextranos de interés para la industria alimentaria a partir del jugo de caña de azúcar.

\section{AGRADECIMIENTOS}

A la Universidad Nacional de Jaén por su interés en publicar los trabajos de investigación de sus docentes y a mi familia por su apoyo constante y sincero.

\section{REFERENCIAS BIBLIOGRÁFICAS}

- Behravan J, B. Sedigheh, S. Zohreh. 2003. Optimization production by Leuconostoc mesenteroides NRRL B-512 using cheap and local sources of carbohydrate and nitrogen. Biotecnhol Appl. Biochem 38:267-269.
- Domínguez L, G. Michelena, E. Carrera. 2004. Biorreactor de dextransucrasa inmovilizada para la obtención de dextranas de bajo peso molecular. Vol. 21, No 01. Departamento de Bioingeniería, Instituto Cubano de Investigaciones de los Derivados de la Caña de Azúcar (ICIDCA). Centro de Ingeniería de Procesos. Instituto Superior Politécnico "José Antonio Echeverría" (ISPJAE). Ciudad de la Habana, Cuba. Biotecnología Aplicada.

- Erhardt F, S. Stammen, H. Jordening. 2008. Production, characterization and coimmobilization of dextranase from Penicillium aculeatum. Biotechnol Lett. Department for Carbohydrate Technology, Institute for Technical Chemistry, Technical University Braunschweig. Germany.

- Hamdy M, E. Gardner, G. Stahly, H. Weiser, Q. Van Winkle. 1954. Factors affecting production and clarification of dextran. Departments of Bacteriology and Chemistry. The Ohio Journal of Science.the Ohio State University, Columbus 10. 54(5): 317.

- Holt J, N. Krieng, N. Sneath, P. Staley, J. Williams. 1994. BergeyS Manual of

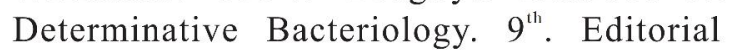
Williams Wilkins. USA.

- Kent J, H. Riegel's. 2007. Handbook of Industrial Chemistry and Biotechnology. Springer. $11^{\text {th }}$. Chapter 30 .

- Landon R, R. Law, C. Webb. 1993. Fermentation broth rheology during dextran production by Leuconostoc mesenteroides BS12(F) as a possible tool for control. Department of Chemical Engincering. University of Manchester Institute of Science and Technology, P.O. Appl Microbiol Biotechnol. Manchester. 40:251-257. 
- Mata J. 2006. Caracterización de los exopolisacáridos producidos por microorganismos halófilos pertenecientes a los géneros Halomonas, Alteromonas, Idiomarinas Palleronia y Salipiger. Tesis Doctoral. Universidad de Granada. Facultad de Farmacia. Departamento de Microbiología.

- Pinchi M, M. Esparza. 2004. Producción de dextranos por Leuconostoc mesenteroides a diferentes tiempos de fermentación usando biorreactores tipo tanque aireado y agitado. Centro de Investigación y Asesoramiento en Biotecnología CIABT - Trujillo. Segunda Semana de Ciencia y Tecnología. Chota-Perú.

- Qaner S, L. Iqbal, L. Rizvi, H. Zuberi. 2001. Producción de dextrano a partir de una Tensión con sacarosa por Leuconostoc mesenteroides PCSIR - 3 Referente a Leuconostoc mesenteroides NRRL B - 512 F. Sección de Biotecnología y Bioquímica. Pharma y Centro Químico de Investigación Multa, P.C.S.I.R. Complejo de Laboratorios, Karachi. Pakistán. 34: $93-97$.

- Qader S, L. Iqbal, A. Aman, E. Shireen, A. Azhar. 2006. Production of Dextran by Newly Isolated Strains of Leuconostoc mesenteroides PCSIR-4 and PCSIR-9. Turkish Journal of Biochemistry - Turk J Biochem. Karachi Pakistan. 31 (1);21-26.

- Rodríguez O, H. Hanssen. 2007. Obtención de dextrano y fructosa, utilizando residuos agroindustriales con la cepa Leuconostoc mesenteroides NRRL B512-F. Revista EIA, ISSN 1794-1237. Escuela de Ingeniería de Antioquia, Medellín. Colombia. Número 7.

- Villanueva C. y C. Carreño. 2005. Manual de Prácticas de Microbiología Industrial. Departamento de Microbiología. Área de Biotecnología. Facultad de Ciencias Biológicas. Universidad Nacional Pedro Ruiz Gallo. Lambayeque. Perú.

\section{Correspondencia:}

Juan Miguel Velásquez Caro

Calle: Toparpa No125 - La Victoria - Chiclayo - Lambayeque Perú

mivelcar@hotmail.com 